\title{
Synthetic vanillate-regulated promoter for graded gene expression in Sphingomonas
}

BACTERIAL TECHNIQUES

AND APPLICATIONS

BACTERIAL GENE

Received

29 July 2014

Accepted

3 September 2014

Published

29 September 2014

Correspondence and requests for materials should be addressed to A.F.-C. (fanne@micro. biol.ethz.ch)

Institute of Microbiology, ETH Zurich, Vladimir-Prelog-Weg 4, 8093 Zurich, Switzerland.

Regulated promoters are an important basic genetic tool allowing, for example, gene-dosage and gene depletion studies. We have previously described a cumate-inducible promoter $\left(\mathrm{P}_{\mathrm{Q} 5}\right)$ that is functional in diverse Alphaproteobacteria. This promoter has been engineered by combining a synthetic minimal promoter, $\mathrm{P}_{\text {syn2 } 2}$, and operator sites and the repressor of the Pseudomonas putida F1 cym/ cmt system. In the present study, we engineered a vanillate-regulated promoter using $P_{\text {syn2 }}$ and the regulatory elements of the Caulobacter crescentus van $R-\operatorname{van} A B$ system. We show that the resulting promoter, which we called $P_{\mathrm{V} 10}$, responds rapidly to the inducer vanillate with an induction ratio of about two orders of magnitude in Sphingomonas melonis Fr1. In contrast to the switch-like behavior of $\mathrm{P}_{\mathrm{Q} 5}, \mathrm{P}_{\mathrm{V} 10}$ shows a linear dose-response curve at intermediate vanillate concentrations, allowing graded gene expression. $P_{V 10}$ is functionally compatible with and independent of $\mathrm{P}_{\mathrm{Q} 5}$ and cumate, and vice versa, suggesting that both systems can be used simultaneously.

egulated promoters are an essential genetic tool for studying bacterial physiology as well as for synthetic biology and industrial applications. For example, they provide means to study essential gene function by depletion analysis and to conditionally express toxic genes. Although multiple of such systems are usually available for a particular model organism, they are often underdeveloped for many non-model organisms, and even constitutive (minimal) promoters are not always available. We have previously described a synthetic approach that allowed us to develop a cumate-inducible expression system that is functional in diverse Alphaproteobacteria, including several sphingomonads, Methylobacterium extorquens and Caulobacter crescen$t_{u s}{ }^{1}$. In this approach, we first identified a minimal promoter consensus based on alignment of several Sphingomonas melonis Fr1 housekeeping gene promoters, then screened for mutations in non-conserved positions in the -10 element of this minimal promoter for increased expression, and finally combined this mutant promoter (termed $\mathrm{P}_{\text {syn2}}$ ) with operator sequences and the repressor of the heterologous $\mathrm{cym} / \mathrm{cmt}$ system, which naturally controls cumate and cymene catabolism in Pseudomonas putida $\mathrm{F} 1^{2,3}$. This engineered promoter (called $\mathrm{P}_{\mathrm{Q} 5}$ ) was cumate-regulated and resulted in induction ratios of two- to three orders of magnitude in the different organisms tested. For sphingomonads, a group of bacteria with great potential in bioremediation, industrial biotechnology and plant protection ${ }^{4-9}$, this was the first dedicated inducible gene expression system described to date.

Because certain applications call for more than one inducible promoter, we wondered whether it would be possible to combine $\mathrm{P}_{\text {syn2 } 2}$ with yet other heterologous operator sequences and repressors so it would be regulated by a stimulus other than cumate. Here we describe such a promoter, termed $\mathrm{P}_{\mathrm{V} 10}$, that combines $\mathrm{P}_{\text {syn2 }}$ with the van $O$ operator sequences of the $v a n A B$ operon and the vanillate-responsive repressor VanR naturally involved in vanillate degradation in the freshwater bacterium Caulobacter crescentus ${ }^{10}$. Our experiments demonstrate that $\mathrm{P}_{\mathrm{V} 10}$ is vanillate-inducible and shows a high dynamic range of gene expression in S. melonis Fr1, and that $\mathrm{P}_{\mathrm{V} 10}$ and $\mathrm{P}_{\mathrm{Q} 5}$ are orthogonal, with each promoter only responding to its designated stimulus, vanillate and cumate, respectively.

\section{Results}

Design of $\mathbf{P}_{\mathbf{V 1 0}}$. A scheme of the organization of vanR and $\mathrm{P}_{\mathrm{V} 10}$ is shown in Fig. 1a. The design rationale is described in the following. In Caulobacter crescentus, van $A B$ is divergently transcribed from vanR, encoding the GntR-type transcriptional repressor of the $v a n A B$ operon. The $v a n A B$ promoter ( $v a n A B \mathrm{p}$ ) has been mapped and shows -35 (TTGACG) and -10 (AAGATT) boxes indicative of a housekeeping, $\sigma^{70}$-dependent promoter ${ }^{10}$. 

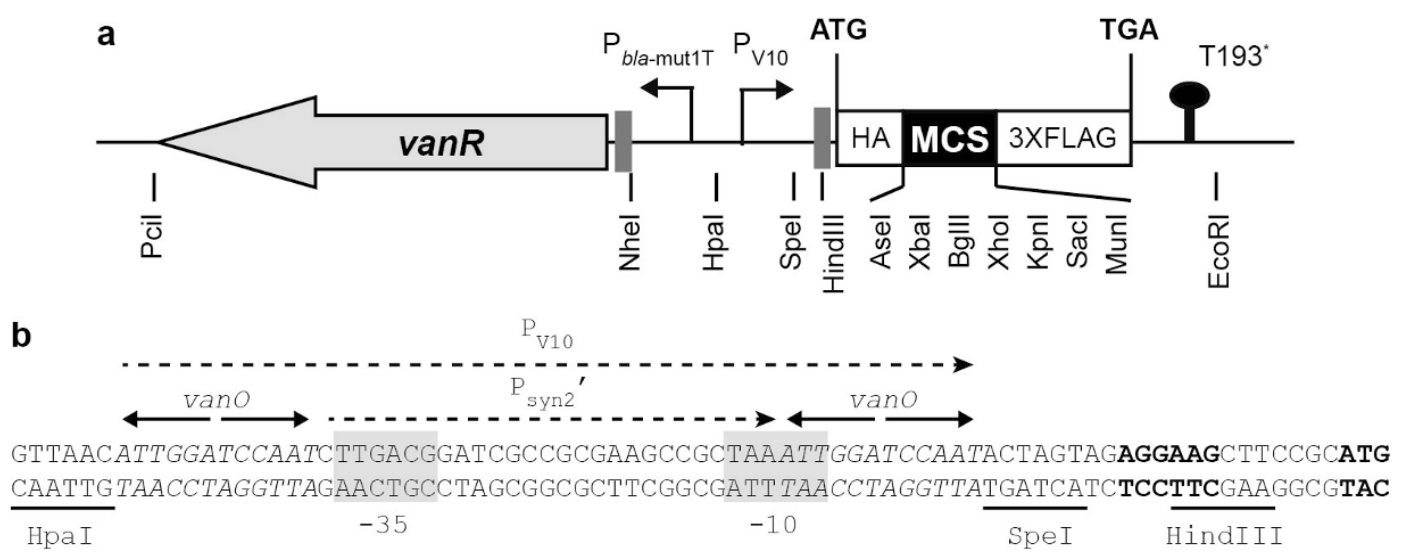

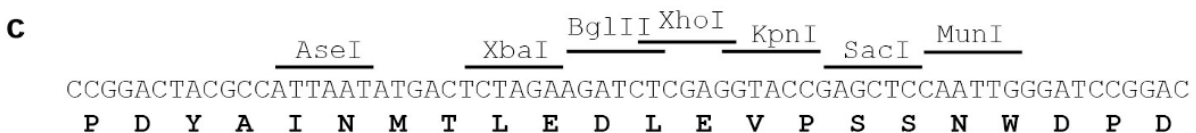

Figure 1 Genetic organization and nucleotide sequence of $\mathrm{P}_{\mathrm{V} 10}$. (a) Organization of $v a n R, \mathrm{P}_{\mathrm{V} 10}$ and the multiple cloning site (MCS) on plasmid $\mathrm{pVH}$. Bended arrows denote promoters and dark grey boxes indicate ribosome bind sites (RBS). Start and stop codons of the open reading frame encoding HA and 3XFLAG tags (white boxes) up- and downstream of the MCS (black box) are indicated by ATG and TGA, respectively. T193* denotes a putative transcriptional terminator. Unique restriction sites in $\mathrm{pVH}$ are shown. (b) Nucleotide sequence of $\mathrm{P}_{\mathrm{V} 10}$. The $3^{\prime}$-truncated $\mathrm{P}_{\text {syn2 }}$ core promoter $\left(\mathrm{P}_{\text {syn2 }}\right.$; see the main text) containing -35 and -10 boxes (highlighted in light gray) and $\mathrm{P}_{\mathrm{V} 10}$ are indicated by dashed arrows above the nucleotide sequence. Palindromic vanO operator sites are indicated by inverted solid arrows and are highlighted in italics in the nucleotide sequence. The RBS and start codon are in bold. (c) Nucleotide sequence, translation and restriction sites of the MCS.

Two perfect inverted repeats (ATTGGATCCAAT), each comprising one operator site $(\operatorname{vanO})$ to which VanR is thought to bind, are present in the $v a n R-v a n A B$ intergenic region, one immediately upstream of the $v a n A B \mathrm{p}-35$ box and the second overlapping the -10 box (positions -9 to -7 ) and the +1 transcriptional start site ${ }^{10}$. The strong synthetic promoter $\mathrm{P}_{\text {syn2 } 2}$ harbors -35 (TTGACG) and -10 (TAACTGC) boxes characteristic for $\sigma^{70}$-dependent promoters, with positions $-12,-11,-7$ and -6 (underlined) in the -10 box highly conserved in numerous $S$. melonis Fr1 housekeeping promoters ${ }^{1}$. To render $\mathrm{P}_{\text {syn2 }}$ regulated by vanillate, we combined $\mathrm{P}_{\text {syn2 } 2}$ with vanO sites in essentially the same configuration observed in the C. crescentus van $A B$ promoter, and we termed the resulting promoter $P_{V 10}$. This required changing the -10 box from TAACTGC to TAAATTG (Fig. 1b), so that the downstream van $O$ sequence overlaps the -10 box (the modified core promoter is referred to as $\mathrm{P}_{\text {syn2 }}$ ); although this might lead to a weakened promoter (see below), we reasoned that, at the same time, this configuration would allow tight repression. In fact, $\mathrm{P}_{\text {syn } 2}$ is very strong ${ }^{1}$ and such strong expression is probably not needed in most cases where expression levels in the physiological range are desired. $v a n R$ was placed under control of the constitutive promoter

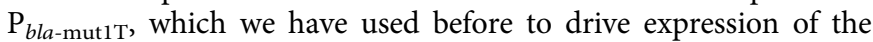
$\mathrm{P}_{\mathrm{Q} 5}$ repressor $\mathrm{CymR}^{* 1}$. The basic plasmid for vanillate-regulated gene expression is $\mathrm{pVH}$, a derivative of the broad-host-range plasmid pCM62 $2^{11}$, in which downstream of $\mathrm{P}_{\mathrm{V} 10}$ a multiple cloning site (Fig. $1 \mathrm{c}$ ) is present that is flanked $5^{\prime}$ by a sequence encoding a hemagglutinin (HA) tag and $3^{\prime}$ by a sequence coding for a triple FLAG (3XFLAG) tag and a putative rho-independent transcriptional terminator (Fig. 1a).

Characterization of $\mathbf{P}_{\mathbf{V 1 0}}$. In order to characterize $\mathrm{P}_{\mathrm{V} 10}$-dependent gene expression in $S$. melonis $\mathrm{Fr} 1, \mathrm{P}_{\mathrm{V} 10}$ was transcriptionally fused to E. coli lacZ (plasmid pVH-lacZ), and $\mathrm{P}_{\mathrm{V} 10^{-}}$lac $\mathrm{Z}^{+}$activity was followed in strain JVZ857/pVH-lacZ grown with different vanillate concentrations using $\beta$-galactosidase assays. As shown in Fig. 2a, $\mathrm{P}_{\mathrm{V} 10}$-lac $Z^{+}$activity was dependent on the inducer concentration, showing a low basal activity (48 $+/-8$ Miller units) without vanillate and high activity (3600 $+/-280$ Miller units) at the highest vanillate concentration tested $(250 \mu \mathrm{M})$. This represents a maximal induction ratio of 74 -fold. In the range of 6.5 to $74 \mu \mathrm{M}$ vanillate, the dose-response curve was essentially linear $\left(\mathrm{R}^{2}=0.994\right)$, indicating that $\mathrm{P}_{\mathrm{V} 10}$ allows graded gene expression, rather than showing switch-like behavior. To follow induction dynamics, JVZ857/pVH-lacZ was grown to mid-exponential phase, $\mathrm{P}_{\mathrm{V} 10}$ was induced by addition of $250 \mu \mathrm{M}$ vanillate and $\mathrm{P}_{\mathrm{V} 10^{-}}$lac $Z^{+}$activity was repeatedly measured over $4.5 \mathrm{~h}$. As seen from Fig. $2 \mathrm{~b}$, the response is rapid and sustained. To see whether $\mathrm{P}_{\mathrm{V} 10}$ could be used simultaneously with the previously characterized cumate-inducible promoter $\mathrm{P}_{\mathrm{Q} 5}$, we tested both promoters for their response to vanillate and/or cumate. Like for $\mathrm{P}_{\mathrm{V} 10}, \mathrm{P}_{\mathrm{Q} 5}$ activity was followed using strain JVZ857 harboring a plasmid-borne $\mathrm{P}_{\mathrm{Q} 5}-$ lac $\mathrm{Z}^{+}$transcriptional fusion described previously ${ }^{1}$. As shown in Fig. $2 \mathrm{c}, \mathrm{P}_{\mathrm{V} 10}$ did not respond to cumate, and cumate had no effect on the induction by vanillate. Similarly, $\mathrm{P}_{\mathrm{Q} 5}$ showed no response to vanillate, and vanillate did not affect the capacity of $\mathrm{P}_{\mathrm{Q} 5}$ to respond to cumate.

In summary, our result demonstrate that $\mathrm{P}_{\mathrm{V} 10}$ is a rapidly responding, vanillate-inducible promoter in S. melonis Fr1. Furthermore, they suggest that $\mathrm{P}_{\mathrm{V} 10}$ is orthogonal to the previously described cumateinducible promoter $\mathrm{P}_{\mathrm{Q} 5}$, and both promoters can be used simultaneously without interference.

Other vanillate-regulated expression plasmids. In addition to the basic vanillate-inducible expression plasmid $\mathrm{pVH}$, we have also constructed two destination plasmids for Gateway cloning, pVHD and pVYD, that allow C-terminal fusions to the HA tag and SYFP2, respectively, and three plasmids for $\mathrm{N}$-terminal fusions to mCherry, SYFP2, and mTq2 (pVCC, pVCY, and pVCTq, respectively). Plasmids will be made available from Addgene (www.addgene.org).

\section{Discussion}

Altogether, our results demonstrate that $\mathrm{P}_{\mathrm{V} 10}$ allows tuning gene expression over a high dynamic range in S. melonis Fr1, and possibly other Alphaproteobacteria. This graded response to vanillate is in contrast to the more switch-like behavior of the previously described cumate-inducible promoter $\mathrm{P}_{\mathrm{Q} 5}{ }^{1}$, at least in S. melonis Fr1, and might make $\mathrm{P}_{\mathrm{V} 10}$ the better choice when gene-dosage should be repro- 
a

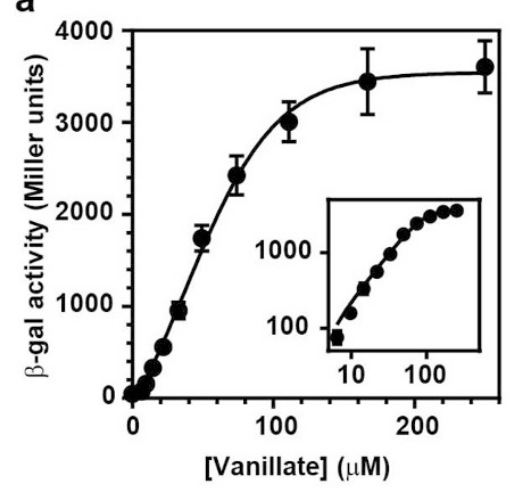

b

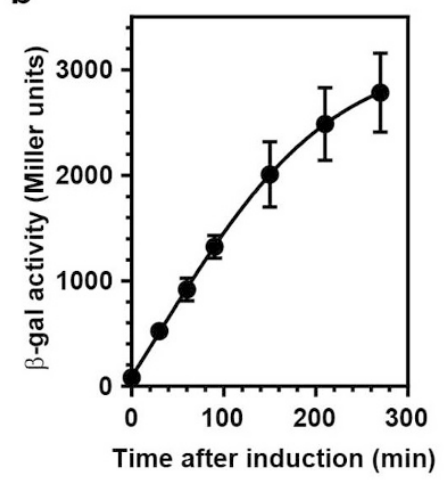

C

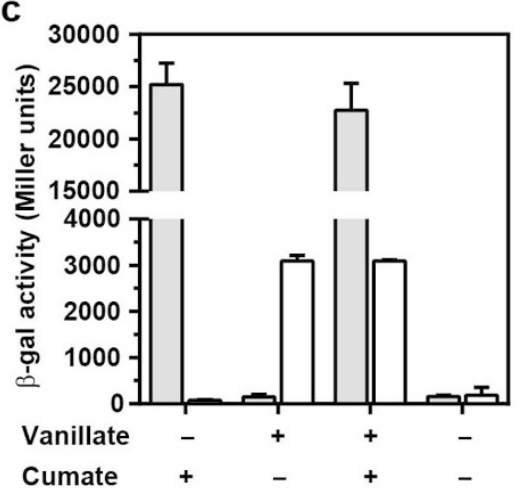

Figure $2 \mid$ Characterization of $\mathrm{P}_{\mathrm{V} 10^{\circ}}$ (a) Dose-response curve of $\mathrm{P}_{\mathrm{V} 10}$ as measured with $\beta$-galactosidase ( $\beta$-gal.) assays on $S$. melonis Fr1 carrying the $\mathrm{P}_{\mathrm{V} 10^{-}}$ lac $Z^{+}$transcriptional fusion (JVZ857/pVH-lacZ). Vanillate concentrations tested ranged from 6.5 to $250 \mu \mathrm{M}$, in 1.5 -fold increments, and no vanillate as control. The inset is a log-log representation of the same data to illustrate induction at low vanillate concentrations. (b) Induction kinetics of $\mathrm{P}_{\mathrm{V} 10}$ were determined by addition of $250 \mu \mathrm{M}$ vanillate to a non-induced culture of JVZ857/pVH-lacZ grown to mid-exponential phase and following $\beta$-gal. activity over time. (c) Strains JVZ857/pVH-lacZ (white bars) and JVZ857/pQF-lacZ carrying the $\mathrm{P}_{\mathrm{Q} 5}$-lacZ $Z^{+}$transcriptional fusion (grey bars) were grown in the presence $(+)$ or absence $(-)$ of $250 \mu \mathrm{M}$ vanillate and/or $25 \mu \mathrm{M}$ cumate as indicated and $\beta$-gal. activities were determined. All data represent the mean $+/-\mathrm{SD}$ of three independent biological replicates.

ducibly and carefully regulated. In contrast, compared to $\mathrm{P}_{\mathrm{V} 10}, \mathrm{P}_{\mathrm{Q} 5}$ shows both higher absolute expression and relative induction upon inducer addition ${ }^{1}$, making $\mathrm{P}_{\mathrm{Q} 5}$ more suitable when very high gene expression levels are desired, e.g. for overexpression studies. Thus, the two promoters are complementary and one or the other might be better suited depending on the biological question. Importantly, because there is no crosstalk between the CymR*/P $\mathrm{P}_{\mathrm{Q} 5}$ and VanR/ $\mathrm{P}_{\mathrm{V} 10}$ systems, i.e. they are orthogonal, both can be used simultaneously, allowing more sophisticated genetic studies of bacterial physiology.

\section{Methods}

Strains and growth conditions. Escherichia coli TOP10 (Invitrogen) or " $c c d B$ survival" (Invitrogen) were used for cloning and routinely grown in LB-Lennox at $37^{\circ} \mathrm{C}$. S. melonis Frl wild-type strain JVZ857 $7^{12}$ was grown in LB-Lennox at $28^{\circ} \mathrm{C}$. Plasmids were transformed in S. melonis by electroporation as previously described ${ }^{13}$. When appropriate, antibiotics were added at the following concentrations: tetracycline $(10 \mu \mathrm{g} / \mathrm{ml})$ and chloramphenicol $(34 \mu \mathrm{g} / \mathrm{ml})$. Vanillate (4-hydroxy-3methoxybezoic acid) was purchased from Sigma-Aldrich (Cat. No. W398802-25G) and dissolved in ethanol to give $1000 \times$ stock solutions for final concentrations indicated in the figure legends. Cumate stocks were prepared as described previously ${ }^{1}$. For "no vanillate" and "no cumate" controls, cultures were mock treated with $0.1 \%$ ( $\mathrm{vol} / \mathrm{vol})$ ethanol.

Plasmid construction. Standard molecular biology protocols were followed ${ }^{14}$. Phusion DNA polymerase for PCR and restriction enzymes were from Thermo Scientific, and T4 DNA ligase was from New England Biolabs. pVH was constructed in two steps. First, $\operatorname{van} R$ was amplified from plasmid pRVYFPC- $2^{10}$ using primers VanR_PciI_F (5' $5^{\prime}$-ATT TAC ATG TTT TCA GTC GGC GCG AAT GC-3') and VanR_NheI_R (5' -ATT TTG CTA GCA GGG AGA GAC CCC GAA TGG ACA TGC CGC GCA TAA- $3^{\prime}$ ) and cloned in $\mathrm{pQH}{ }^{1}$ via PciI/NheI, replacing $c y m R^{*}$. Then, a synthetic fragment (Eurofins, MWG Operon, Germany) containing $\mathrm{P}_{\text {bla-mut1T }}{ }^{1}$ for vanR expression and $\mathrm{P}_{\mathrm{V} 10}$ for vanillate-regulated expression was amplified using primers PV10_F (5'-ATT TGC TAG CAT CAG GGT TAT TG-3') and PV10_R (5' ATT TAA GCT TCC TCT ACT AGT ATT G-3') and cloned between NheI/HindIII. To construct pVH-lacZ, lacZ was excised from pAK127lacZ(MCS) ${ }^{1}$ using XbaI/ EcoRI and cloned in $\mathrm{pVH}$ via SpeI/EcoRI. $\mathrm{pVHD}$ was constructed by amplification of the Gateway cassette from pDEST-565 (Addgene plasmid 11520) using primers oJVZ739 (5'-ATT TGG TAC CTC TAG CTA GCG ATA TCA CC-3') and oJVZ740 (5'-ATT TTC TAG AGA CAA GTT TGT ACA AAA AAG C-3') and cloning in pVH via XbaI/Acc65I. pVYD was obtained by subcloning a PsiI/SpeI fragment of $\mathrm{pVH}$ containing vanR and $\mathrm{P}_{\mathrm{V} 10}$ in between the same sites of $\mathrm{pQYD}^{1}$, replacing $c y m R^{*}$ and $\mathrm{P}_{\mathrm{Q} 5}$. Plasmids $\mathrm{pVCY}, \mathrm{pVCC}$ and $\mathrm{pVCTq}$ were constructed by amplifying the genes encoding fluorescent proteins with primers mTq2C_F (5'-ATT TGG TAC CGA GCT CCA ATT GGG GCG GCG GCA GCG GCG GCG GCA GCG TGA GCA AGG GCG AGG AGC-3') and mTq2C_R (5'-ATT TTG AAT TCT CAC TTG TAC AGC TCG TCC ATG CC- $3^{\prime}$ ), digestion of the PCR product with Acc65I/EcoRI, and cloning in $\mathrm{pVH}$ via Acc65I/MunI. Templates for PCR were: $\mathrm{pQY} \mathrm{Y}^{1}$ for "super" yellow fluorescent protein 2 (SYFP2); $\mathrm{pQR}^{1}$ for mCherry; $\mathrm{pmTurquoise2- \textrm {C } ^ { 1 5 } \text { for }}$ mTurqouise2 (mTq2).
Reporter assays. Promoter activities were measured essentially as described previously ${ }^{1}$. For dose-response curves and cross-induction experiments, S. melonis Fr1 carrying $\mathrm{pVH}$-lacZ or pQF-lacZ ${ }^{1}$ were grown in LB-Lennox containing different concentrations of vanillate and/or cumate overnight to mid-exponential phase and $\beta$ galactosidase activity was measured according to $\mathrm{Miller}^{16}$. To follow induction kinetics, S. melonis carrying $\mathrm{pVH}-\mathrm{lacZ}$ was grown to mid-exponential phase and induced by the addition of $250 \mu \mathrm{M}$ vanillate, and $\beta$-galactosidase was measured at different time points. All results are presented as mean $+/-\mathrm{SD}$ of three biological replicates. Linear regression analysis to evaluate linearity of the dose-response curve was performed in GraphPad Prism 5 (version 5.04, Graphpad Software Inc., USA).

1. Kaczmarczyk, A., Vorholt, J. A. \& Francez-Charlot, A. Cumate-inducible gene expression system for sphingomonads and other Alphaproteobacteria. Appl. Environ. Microbiol. 79, 6795-6802 (2013).

2. Eaton, R. W. p-Cumate catabolic pathway in Pseudomonas putida Fl: cloning and characterization of DNA carrying the cmt operon. J. Bacteriol. 178, 1351-1362 (1996).

3. Eaton, R. W. p-Cymene catabolic pathway in Pseudomonas putida F1: Cloning and characterization of DNA encoding conversion of $p$-cymene to $p$-cumate. J. Bacteriol. 179, 3171-3180 (1997).

4. Balkwill, D. L., Fredrickson, J. K. \& Romine, M. F. in The Prokaryotes: A Handbook on the Biology of Bacteria, Vol.7, Proteobacteria, Delta and Epsilon Subclasses. Deeply Rooting Bacteria. 605-629 (Springer-SBM, New York, 2006).

5. Fialho, A. M. et al. Occurrence, production, and applications of gellan: current state and perspectives. Appl. Microbiol. Biotechnol. 79, 889-900 (2008).

6. Innerebner, G., Knief, C. \& Vorholt, J. A. Protection of Arabidopsis thaliana against leaf-pathogenic Pseudomonas syringae by Sphingomonas strains in a controlled model system. Appl. Environ. Microbiol. 77, 3202-3210 (2011).

7. Takeuchi, M., Hamana, K. \& Hiraishi, A. Proposal of the genus Sphingomonas sensu stricto and three new genera, Sphingobium, Novosphingobium and Sphingopyxis, on the basis of phylogenetic and chemotaxonomic analyses. Int. J. Syst. Evol. Microbiol. 51, 1405-1417 (2001).

8. Vogel, C., Innerebner, G., Zingg, J., Guder, J. \& Vorholt, J. A. Forward genetic in planta screen for identification of plant-protective traits of Sphingomonas sp. strain Frl against Pseudomonas syringae DC3000. Appl. Environ. Microbiol. 78, 5529-5535 (2012).

9. White, D. C., Sutton, S. D. \& Ringelberg, D. B. The genus Sphingomonas: physiology and ecology. Curr. Opin. Biotechnol. 7, 301-306 (1996).

10. Thanbichler, M., Iniesta, A. A. \& Shapiro, L. A comprehensive set of plasmids for vanillate- and xylose-inducible gene expression in Caulobacter crescentus. Nucleic Acids Res. 35, e137 (2007)

11. Marx, C. J. \& Lidstrom, M. E. Development of improved versatile broad-hostrange vectors for use in methylotrophs and other Gram-negative bacteria. Microbiology 147, 2065-2075 (2001).

12. Kaczmarczyk, A. et al. Role of Sphingomonas sp. strain Fr1 PhyR-NepR- $\sigma^{\mathrm{EcfG}}$ cascade in general stress response and identification of a negative regulator of PhyR. J. Bacteriol. 193, 6629-6638 (2011).

13. Kaczmarczyk, A., Vorholt, J. A. \& Francez-Charlot, A. Markerless gene deletion system for sphingomonads. Appl. Environ. Microbiol. 78, 3774-3777 (2012).

14. Sambrook, J. \& Russel, D. Molecular Cloning: A Laboratory Manual. Third edn, (Cold Spring Harbor Laboratory Press, Cold Spring Harbor, NY, 2001). 
15. Goedhart, J. et al. Structure-guided evolution of cyan fluorescent proteins towards a quantum yield of 93\%. Nature communications 3, 751 (2012).

16. Miller, J. H. Experiments in Molecular Genetics. 352-355 (Cold Spring Harbor Laboratory Press, Cold Spring Harbor, NY, 1972).

\section{Acknowledgments}

We thank Martin Thanbichler, Dominic Esposito, Joachim Goedhart and Theodorus W. J. Gadella for plasmids. This work was supported by Swiss National Science Foundation (SNF) grant 31003B-152835.

\section{Author contributions}

A.K. designed and performed experiments. A.K., J.A.V. and A.F.-C. conceived the project and wrote the manuscript.

\section{Additional information}

Competing financial interests: The authors declare no competing financial interests.

How to cite this article: Kaczmarczyk, A., Vorholt, J.A. \& Francez-Charlot, A. Synthetic vanillate-regulated promoter for graded gene expression in Sphingomonas. Sci. Rep. 4, 6453; DOI:10.1038/srep06453 (2014).

This work is licensed under a Creative Commons Attribution-NonCommercialNoDerivs 4.0 International License. The images or other third party material in this article are included in the article's Creative Commons license, unless indicated otherwise in the credit line; if the material is not included under the Creative Commons license, users will need to obtain permission from the license holder in order to reproduce the material. To view a copy of this license, visit http:// creativecommons.org/licenses/by-nc-nd/4.0/ 